\title{
An inflammation-based cumulative prognostic score system in patients with diffuse large $B$ cell lymphoma in rituximab era
}

Feifei Sun ${ }^{1,2+}$, Jia Zhu ${ }^{1,2 \dagger}$, Suying Lu $u^{1,2}$, Zijun Zhen ${ }^{1,2}$, Juan Wang ${ }^{1,2}$, Junting Huang ${ }^{1,2}$, Zonghui Ding ${ }^{3}$, Musheng Zeng ${ }^{1 *}$ and Xiaofei Sun ${ }^{1,2^{*}}$

\begin{abstract}
Background: Systemic inflammatory parameters are associated with poor outcomes in malignant patients. Several inflammation-based cumulative prognostic score systems were established for various solid tumors. However, there is few inflammation based cumulative prognostic score system for patients with diffuse large B cell lymphoma (DLBCL).

Methods: We retrospectively reviewed 564 adult DLBCL patients who had received rituximab, cyclophosphamide, doxorubicin, vincristine and prednisolone (R-CHOP) therapy between Nov 12006 and Dec 302013 and assessed the prognostic significance of six systemic inflammatory parameters evaluated in previous studies by univariate and multivariate analysis:C-reactive protein(CRP), albumin levels, the lymphocyte-monocyte ratio (LMR), the neutrophillymphocyte ratio(NLR), the platelet-lymphocyte ratio(PLR)and fibrinogen levels.

Results: Multivariate analysis identified CRP, albumin levels and the LMR are three independent prognostic parameters for overall survival (OS). Based on these three factors, we constructed a novel inflammation-based cumulative prognostic score (ICPS) system. Four risk groups were formed: group ICPS $=0$, ICPS $=1$, ICPS $=2$ and ICPS $=3$. Advanced multivariate analysis indicated that the ICPS model is a prognostic score system independent of International Prognostic Index (IPI) for both progression-free survival (PFS) $(p<0.001)$ and OS $(p<0.001)$. The 3 -year OS for patients with ICPS $=0, I C P S=1$, ICPS $=2$ and ICPS $=3$ were $95.6,88.2,76.0$ and $62.2 \%$, respectively $(p<0.001)$. The 3 -year PFS for patients with ICPS $=$ $0-1$, ICPS $=2$ and ICPS $=3$ were 84.8, 71.6 and $54.5 \%$, respectively $(p<0.001)$.

Conclusions: The prognostic value of the ICPS model indicated that the degree of systemic inflammatory status was associated with clinical outcomes of patients with DLBCL in rituximab era. The ICPS model was shown to classify risk groups more accurately than any single inflammatory prognostic parameters. These findings may be useful for identifying candidates for further inflammation-related mechanism research or novel anti-inflammation target therapies.
\end{abstract}

Keywords: Diffuse large B-cell lymphoma, Rituximab, Inflammation, Score system, Prognostic

\footnotetext{
* Correspondence: zengmsh@sina.com; sunxf@sysucc.org.cn

${ }^{\dagger}$ Equal contributors

${ }^{1}$ State Key Laboratory of Oncology in South China, Collaborative Innovation

Center for Cancer Medicine, Sun Yat-sen University Cancer Center, N0.651 of

Dongfeng East Road, Guangzhou 510060, China

Full list of author information is available at the end of the article
} 


\section{Background}

Diffuse large B-cell lymphoma (DLBCL) is the most common subtype of non-Hodgkin lymphoma (NHL), representing $30-40 \%$ of all lymphomas. It is an aggressive lymphoma with heterogeneous clinicopathological, immunephenotypic, genetic features and various clinical outcomes [1-4]. Although the addition of rituximab (R) to conventional $\mathrm{CHOP}$ or $\mathrm{CHOP}$-like regimens has significantly improved survival, approximately $30 \%$ of DLBCL patients fail chemotherapy [5]. The International Prognostic Index (IPI) based on age, performance status (PS), Ann Arbor stage, number of extranodal lesions and serum lactate dehydrogenase (LDH) level is a standard prognostic scoring system for predicting the clinical outcomes of patients with DLBCL. But in rituximab era, the ability of the IPI to predict prognosis has declined [6]. A variety of molecular biomarkers and gene signatures with prognostic significance have been identified in DLBCL patients [1-4]. However, molecular markers and gene signatures are expensive, technically challenging, and not routinely available in many undeveloped countries. Therefore, cheap and easily accessible prognostic markers which might help to increase prognostic accuracy are needed.

Malignant and inflammation are closely linked. Inflammatory processes have been identified to play an important role in the pathogenesis of lymphoma [7-9]. Pro-inflammatory cytokines (or chemokines) and inflammatory cells and in tumor microenvironment were proved to promote tumor growth, DNA damage, angiogenesis, immune suppression and to be associated with poor survival outcomes of patients [10-13]. Cytokine receptors or other factors in inflammatory pathways implicated in metastasis may be an appropriate target for malignant tumor therapy [14-19]. Circulating inflammatory parameter(including C-reactive protein (CRP), albumin levels, the lymphocyte-monocyte ratio (LMR), the neutrophil-lymphocyte ratio (NLR), the platelet-lymphocyte ratio (PLR) or fibrinogen levels) was associated with a poor prognosis in many types of malignant tumor patients including DLBCL [1, 20-27]. Several inflammation-based cumulative prognostic score systems were established for various solid tumors [28-31], but there is few inflammation-based cumulative prognostic score system for predicting survival of patients with diffuse large $B$ cell lymphoma. Therefore, this retrospective study aimed to develop a novel inflammation-based cumulative prognostic score system for predicting survival of patients with diffuse large B cell lymphoma in rituximab era. The system may be useful for identify candidates for further inflammation related research and clinical trial of novel anti-inflammation drug.

\section{Methods}

\section{Patients}

We reviewed the records of 839 patients diagnosed with DLBCL according to the 2008 World Health Organization (WHO) criteria [32] at the Sun Yat-Sen University Cancer Center of China between November 1, 2006 and December 30, 2013. The data for 564 patients who received $\mathrm{R}-\mathrm{CHOP}$ therapy as first-line treatment were analyzed. Clinicopathological parameters included gender, age, Ann Arbor stage, number of extranodal sites, Eastern Cooperative Oncology Group performance status (ECOG PS) and LDH level. Ann Arbor stage was made according bone marrow findings, computed tomography (CT) scans of the thorax, abdomen, and pelvic cavity, or whole body positron emission tomography/computed tomography (PET/CT) scans before treatment. The IPI was evaluated based on Ann Arbor stage, ECOG PS, serum LDH and numbers of extranodal sites. The laboratory data, including lymphocyte, neutrophil and platelet counts were obtained 1-3 days before first chemotherapy. Serum levels of CRP, albumin and fibrinogen were obtained 1-7 days before first chemotherapy. The exclusion criteria included: 1.patients treated with chemotherapy other than R-CHOP as first-line therapy; 2.patients with primary central nervous system (CNS) lymphoma; 3.patients with immunodeficiency virus infection; 4.Patients whose dose was reduced $>20 \%$; 5.Patients who did not complete their course of R-CHOP; 6.patients with clinical evidence of acute infection or chronic inflammatory disease. A total of 275 cases were excluded, including 14 cases with primary CNS DLBCL, 200 cases who received chemotherapy regimens other than $\mathrm{R}-\mathrm{CHOP}$ for first-line therapy, 15 cases who did not complete their course of R-CHOP treatment, 3 cases whose therapy dose was reduced $>20 \%$ and 43 cases with acute infection or chronic inflammatory disease.

\section{Treatment}

Patient received standard R-CHOP therapy as firstline treatment $\left[375 \mathrm{mg} / \mathrm{m}^{2}\right.$ rituximab on day 1 , $750 \mathrm{mg} / \mathrm{m}^{2}$ cyclophosphamide on day $2,50 \mathrm{mg} / \mathrm{m}^{2}$ doxorubicin on day 2 , and $1.4 \mathrm{mg} / \mathrm{m}^{2}$ (maximum $2.0 \mathrm{mg} /$ body) vincristine on day 2 and $100 \mathrm{mg} / \mathrm{d}$ prednisolone on days 2-6 for 21 days per cycle] for all DLBCL patients regardless of Ann Arbor stage. Patients who had disease progression at any time or who did not achieve partial response after 4 cycles received salvage therapy. Patients in this group received R-CHOP therapy for 2 to 8 cycles (the median was 6 cycles) as first-line treatment. Residual disease, extranodal disease, or primary bulky disease was treated by radiotherapy followed by chemotherapy. 


\section{Statistical analysis}

PFS was defined from the date of diagnosis to first lymphoma progression or death from any cause, or censored at the date of last follow-up for the patients who were alive and did not have lymphoma progression. OS was defined from the date of diagnosis to death from any cause, or censored at the date of last follow-up for the patients who were alive. The optimal cut-off values for six biomarkers (CRP, albumin levels, LMR, NLR, LPR and fibrinogen levels) for predicting OS were determined using time-dependent operating characteristic (ROC) analysis, which was performed by 'survival ROC package' in R, version 3.3.3 (http://www.r-project.org/). The primary end point was OS, predict.time $=3$ years, and the maximal Youden index (Youden index $=$ sensitivity + specificity-1) was chosen to set optimal cut-off value. Other statistical analysis was performed with SPSS 17.0 software. The log-rank test was used to assess univariate associations between prognostic parameters and survival. The Cox proportional hazards model was used for multivariate analysis. A $P$ value of less than 0.05 was regarded as statistically significant. Survival curves were constructed using the Kaplan-Meier method.

\section{Results}

\section{Patients' characteristics}

A total of 564 newly diagnosed DLBCL patients who received R-CHOP regimens were analyzed, included $381(67.6 \%)$ male and $183(32.4 \%)$ female with a median age of 53 years (range 18-88 years). The Ann Arbor tumor stage distribution was as follows: stage I: 114 (20.2\%) patients, stage II: $196(34.8 \%)$ patients, stage III: 129(22.9\%) patients and stage IV: $125(22.2 \%)$ patients. After a median follow-up time of 31.5 months, 139 patients relapsed or progressed in total and 96 patients died during or after first-line chemotherapy. Patients' characteristics are summarized in Table 1 .

\section{Cut-off values}

First, we conducted ROC analysis to determine the optimal cut-off values of all inflammatory parameters for predicting OS as described in method above. The optimal cut-off value for CRP was $8.6 \mathrm{mg} / \mathrm{L}$ (sensitivity $66.99 \%$ and specificity $67.07 \%$, AUC value $0.703,95 \%$ CI $0.649-0.760, P<0.001)$. The optimal cut-off value of the albumin levels was $41.5 \mathrm{~g} / \mathrm{L}$ (sensitivity $75.78 \%$ and specificity $58.61 \%$, AUC value $0.682,95 \%$ CI $0.614-0.731, P$ $<0.001)$. The optimal cut-off value for the LMR was 2.7 (sensitivity $68.21 \%$ and specificity $67.96 \%$, AUC value $0.704,95 \%$ CI $0.644-0.759, P<0.001)$. The optimal cutoff value for the NLR was 4.6 (sensitivity $44.37 \%$ and specificity $82.75 \%$, AUC value $0.64495 \%$ CI $0.574-0.700$, $P<0.001)$. The optimal cut-off value for the PLR was 187.4(sensitivity $46.20 \%$ and specificity $72.30 \%$, AUC
Table 1 Univariate analysis of clinical parameters

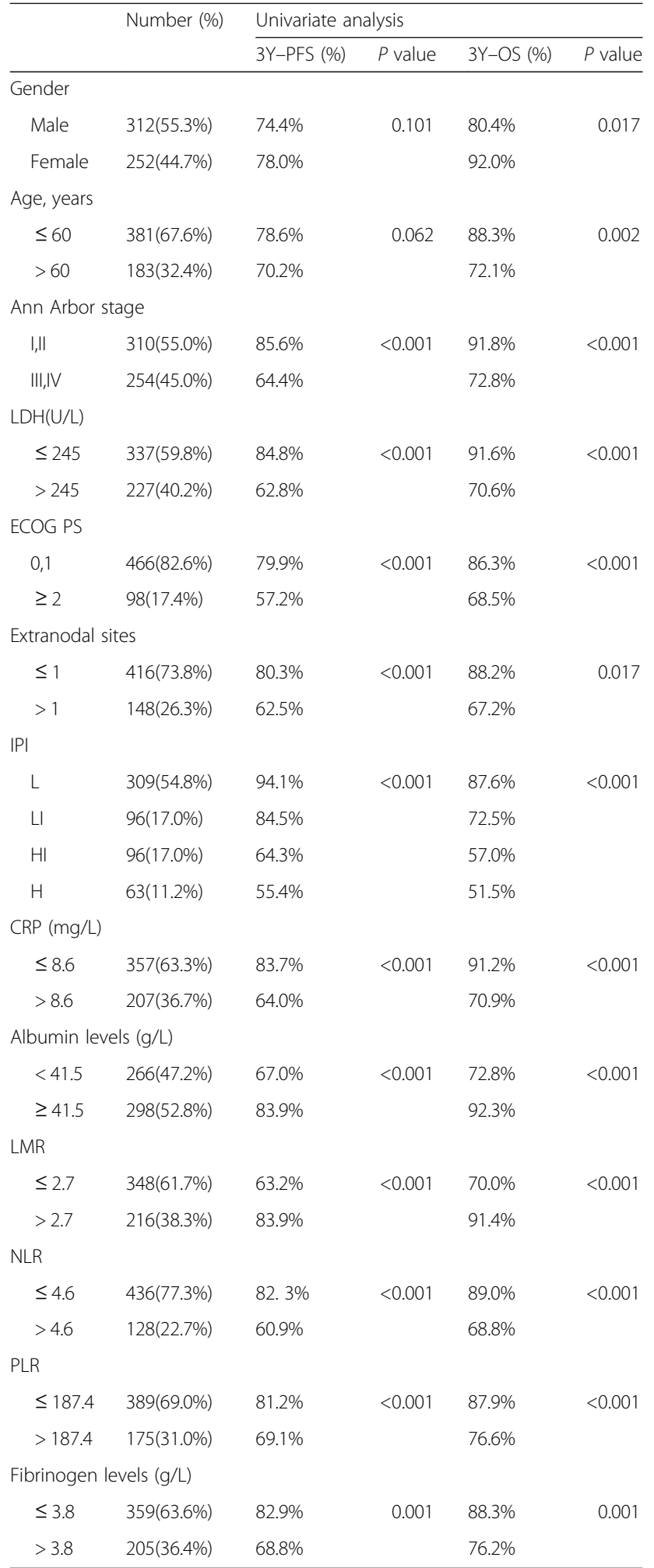

Abbreviations: 3Y-PFS 3-year progression-free survival, 3Y-OS 3-year overall survival, $L D H$ Lactate dehydrogenase, ECOG PS Eastern Cooperative Oncology Group performance status, IPI International Prognostic Index, L Low, LI Low-intermediate, $H I$ High-intermediate, $H$ High, CRP C-reactive protein, $L M R$ Lymphocyte-to-monocyte Ratio, NLR Neutrophil-to- lymphocyte ratio, PLR Platelet-to- lymphocyte ratio 
Table 2 Multivariate analysis of various inflammatory parameters

\begin{tabular}{|c|c|c|c|c|}
\hline \multirow[t]{2}{*}{ Inflammatory parameters } & \multicolumn{2}{|l|}{ OS } & \multicolumn{2}{|l|}{ PFS } \\
\hline & $\mathrm{HR}(95 \% \mathrm{Cl})$ & $P$-value & $\mathrm{HR}(95 \% \mathrm{Cl})$ & $P$-value \\
\hline Albumin levels(<41.5 g/L) & $2.911(1.533-5.525)$ & 0.001 & $2.025(1.278-3.208)$ & 0.003 \\
\hline $\operatorname{LMR}(\leq 2.7)$ & $1.966(1.100-3.513)$ & 0.022 & $1.688(1.062-2.684)$ & 0.027 \\
\hline $\mathrm{CRP}(>8.6 \mathrm{mg} / \mathrm{L})$ & $1.804(0.983-3.310)$ & 0.037 & $1.588(0.993-2.541)$ & 0.054 \\
\hline $\operatorname{NLR}(>4.6)$ & & 0.306 & & 0.183 \\
\hline $\operatorname{PLR}(>187.4)$ & & 0.604 & & 0.205 \\
\hline Fibrinogen levels (>3.8 g/L) & & 0.800 & & 0.266 \\
\hline
\end{tabular}

Abbreviations: OS Overall survival, PFS Progression-free survival, HR Hazard Ratio, 95\% Cl, 95\% confidence limits, LMR Lymphocyte-to-monocyte Ratio, CRP C-reactive protein, NLR Neutrophil-to- lymphocyte ratio, PLR Platelet-to- lymphocyte ratio

value $0.587,95 \%$ CI $0.520-0.652, P=0.011)$. The optimal cut-off value for the fibrinogen was $3.8 \mathrm{~g} / \mathrm{L}$ (sensitivity $54.20 \%$ and specificity $69.20 \%$, AUC value $0.601,95 \% \mathrm{Cl}$ $0.529-0.685, P=0.007)$.

\section{The novel inflammation-based cumulative prognostic score system (ICPS)}

In a univariate analysis, sex, age, localized/advanced stage, EN number, LDH, ECOG PS, IPI risk groups, CRP, albumin levels, LMR, NLR, LPR and fibrinogen levels were all significantly associated with PFS and OS (Table 1). Sex was significantly associated with OS but not PFS. Multivariate cox regression analysis for various inflammatory parameters showed that CRP, albumin levels and LMR were independent risk factors for OS, while NLR, LPR and fibrinogen levels were not (Table 2). Based on these three independent inflammatory adverse factors $(\mathrm{CRP}>8.6 \mathrm{mg} / \mathrm{L}$, albumin $<41.5 \mathrm{~g} / \mathrm{L}$ and LMR $\leq 2.7$ ), we constructed a novel inflammatorybased prognostic model by combing these three prognostic variables. Table 3 shows the hazard ratio (HR) and regression coefficient $(\beta)$ for OS of each significant inflammatory marker. The regression coefficient $(\beta)$ for OS of each significant inflammatory marker was calculated from the Cox regression eq. $\left(H R=e^{\beta}\right)$. Because the HR for OS of each significant inflammatory marker is very close, we put the same weight on each factor in the prognostic-score model. The sum of the points allotted correlates with the following

Table 3 The hazard ratio (HR) and regression coefficient $(\beta)$ for OS of each significant inflammatory marker

\begin{tabular}{llll}
\hline Factor & $\mathrm{HR}$ & $\beta\left(\mathrm{HR}=\mathrm{e}^{\beta}\right)$ & score \\
\hline Albumin levels $<41.5 \mathrm{~g} / \mathrm{L}$ & $2.245(1.366-3.691)$ & 0.81 & 1 \\
$\mathrm{LMR} \leq 2.7$ & $2.019(1.225-3.328)$ & 0.70 & 1 \\
$\mathrm{CRP}>8.6 \mathrm{mg} / \mathrm{L}$ & $1.842(1.102-3.078)$ & 0.61 & 1 \\
\hline
\end{tabular}

Abbreviations: OS Overall survival, HR Hazard Ratio, LMR Lymphocyte-tomonocyte Ratio, CRP C-reactive protein risk groups: group ICPS $=0 \quad(n=202,35.8 \%)$, no inflammatory adverse factors; group ICPS $=1 \quad(n=144$, 25.5\%), 1 factor; group ICPS $=2(n=99,17.6 \%), 2$ factors; and ICPS $=3(n=119,21.1 \%), 3$ factors. In an advanced multivariate analysis adjusted for IPI risk factors (age, localized/advanced stage, EN number, LDH and ECOG PS), ICPS is an independent prognostic factor for both PFS and OS (Table 4). And ICPS is also a prognostic score system independent of IPI score (Table 5). The proportion of patients in each ICPS group and the associated hazard ratios in survival analysis are presented in Table 6 . The relative risk of group ICPS $=0$, ICPS $=1$, ICPS $=2$ and ICPS $=3$ for OS was $1.000,2.270(1.063-4.847), 4.395(2.118-9.118)$ and 8.645(4.482-16.676), respectively. The relative risk of group ICPS $=0$, ICPS $=1$, ICPS $=2$ and ICPS $=3$ for PFS was $1.000,1.409(0.838-2.369), 2.241(1.333-3.767)$ and 3.957(2.518-6.216), respectively. Figures 1 and 2 shows Kaplan-Meier curves for OS and PFS stratified according to the ICPS group, respectively. The 3-year OS rates for patients with ICPS $=0$, ICPS $=1$, ICPS $=2$ and ICPS $=3$ were 95.6, 88.2, 76.0 and $62.2 \%$, respectively(Fig. 1). It showed that patients with lower ICPS had significantly better OS $(p<0.001)$. The ICPS model can significantly distinguish between any neighboring two risk groups. The 3-year PFS rates for patients with ICPS $=0$, ICPS $=1$, ICPS $=2$ and ICPS $=$ 3 were 86.5, 82.3, 71.6 and $54.5 \%$, respectively (Table 6, Fig. 2a). The result showed that the PFS difference between group ICPS $=0$ and ICPS $=1$ is insignificance, but the difference between any two group of ICPS = 0 , ICPS $=2$ and ICPS $=3$ is significant. So we merged patients with ICPS $=0$ and ICPS $=1$ to one group (ICPS $=0-1)$. In PFS analysis, we could classify patients to three risk groups (group ICPS $=0-1$, ICPS $=$ 2 and ICPS $=3$ ) (Fig. 2b). The 3-year PFS rates for patients with ICPS $=0-1, \mathrm{ICPS}=2$ and ICPS $=3$ were $84.8,71.6$ and $54.5 \%$, respectively. The PFS difference between any two of three risk groups is significant ( $p$ $<0.001$ ). 
Table 4 Multivariate analysis of the inflammation-based cumulative prognostic score (ICPS) and IPI risk factors

\begin{tabular}{|c|c|c|c|c|}
\hline \multirow[t]{2}{*}{ Characteristic } & \multicolumn{2}{|l|}{ OS } & \multicolumn{2}{|l|}{ PFS } \\
\hline & $P$-value & HR $(95 \% \mathrm{Cl})$ & $P$-value & HR $(95 \% \mathrm{Cl})$ \\
\hline Age(>60y) & $<0.001$ & $2.493(1.657-3.749)$ & 0.250 & $1.228(0.865-1.745$ \\
\hline Ann Arbor stage(III,IV) & 0.533 & $1.185(0.695-2.022)$ & 0.295 & 1.254(0.821-1.914 \\
\hline $\mathrm{LDH}(>245 \mathrm{U} / \mathrm{L})$ & 0.042 & $1.690(1.020-2.800)$ & 0.018 & $1.642(1.087-2.479$ \\
\hline ECOG PS( $(\geq 2)$ & 0.411 & $1.230(0.751-2.012)$ & 0.022 & $1.579(1.069-2.330$ \\
\hline Extranodal sites $(>1)$ & 0.013 & $1.803(1.134-2.868)$ & 0.187 & 1.305(0.879-1.937) \\
\hline$I C P S \geq 2$ & 0.001 & $2.476(1.483-4.136)$ & 0.019 & $1.611(1.080-2.404$ \\
\hline
\end{tabular}

Abbreviations: OS Overall survival, PFS Progression-free survival, HR Hazard Ratio, 95\% Cl, 95\% confidence limits, LDH Lactate dehydrogenase, ECOG PS Eastern Cooperative Oncology Group performance status, IPI International Prognostic Index, Hi High-intermediate, $H$ High, ICPS Inflammation-based cumulative prognostic score

\section{Discussion}

It is well established that systemic inflammation status is associated with poor outcome in various solid tumors and lymphomas [5, 10, 33-36]. Prognostic parameters which reflect systemic inflammatory response include CRP, albumin levels, LMR, NLR, PLR and fibrinogen levels [1, 20-27], etc. Furthermore, recent studies had indicated that inflammation-based cumulative prognostic score systems, like the Glasgow prognostic score (GPS) or advance lung cancer inflammation index (ALI), are useful prognostic models for several solid tumors [28-31]. A retrospective study aslo showed that the modified Glasgow prognostic scores (mGPS) can be used as a predictor in diffuse large B cell lymphoma treated with R-CHOP regimen [37]: patients with lower mGPS had higher complete response rate and better OS, however, the difference of PFS was statistically insignificant. So far, there is few inflammation-based cumulative prognostic score systems for predicting survival of patients with diffuse large B cell lymphoma. To overcome the lacking data, we accessed prognostic values of inflammatory prognostic parameters commonly used in publications including CRP, albumin levels, LMR, NLR, PLR and fibrinogen levels. The results of univariate and multivariate analysis showed that albumin levels, CRP and LMR are three independent prognostic factors for OS. Based on these three independent inflammatory adverse factors $(\mathrm{CRP}>8.6 \mathrm{mg} / \mathrm{L}$, albumin levels $<41.5 \mathrm{~g} / \mathrm{L}$

Table 5 Multivariate analysis of the inflammation-based cumulative prognostic score (ICPS) and IPI score

\begin{tabular}{llllll}
\hline Characteristic & OS & & & \\
\cline { 2 - 3 } \cline { 5 - 6 } & & $P$-value & HR $(95 \% \mathrm{Cl})$ & & \\
\hline IPI $(\mathrm{Hi}, \mathrm{H})$ & $<0.001$ & $2.697(1.652-4.405)$ & & 0.001 & $1.892(1.293-1.767)$ \\
ICPS $\geq 2$ & $<0.001$ & $3.044(1.929-4.804)$ & & $<0.001$ & $2.293(1.577-3.335)$ \\
\hline
\end{tabular}

Abbreviations: OS Overall survival, PFS Progression-free survival, $H R$ Hazard Ratio, 95\% Cl, 95\% confidence limits, IPI International Prognostic Index, Hi Highintermediate, $H$ High, ICPS Inflammation-based cumulative prognostic score and LMR $\leq 2.7$ ), we developed a novel systemic inflammatory cumulative prognostic score system, which we called the ICPS. An advanced multivariate analysis showed that the ICPS was a prognostic system independent of IPI risk factors including age, Ann Arbor stage, number of extranodal sites, ECOG PS and serum LDH level. Compared with any single inflammatory prognostic parameters, the ICPS model was shown to classify risk groups more accurately. The ICPS model can classify patients to four risk groups for OS and three risk groups for PFS. The survival difference between any neighboring two risk groups is significant in both PFS and OS analysis. As our best knowledge, it is the best inflammatory-based prognostic score system reported in DLBCL patients so far. Patients with higher ICPS had poorer clinical outcomes, which indicated that the degree of systemic inflammatory status had indeed been associated with clinical outcomes of patients with DLBCL in rituximab era. Inflammation plays an important role in malignant tumor development. Inflammatory cytokines (including tumor necrosis factor (TNF), Interleukins (IL) and chemokines, etc.) and immune cells (including tumor-associated macrophages and tumor infiltrating lymphocytes, etc.) in tumor microenvironment contribute to tumor growth, metastasis and immunosuppression [10-13]. Functional polymorphisms of inflammatory cytokine genes have been demonstrated to be associated with lymphoma risk and outcomes [3840]. Chemokines or other inflammatory factors implicated in metastasis are considered to be potential targets for malignant tumor therapy [41-43]. Elevated levels of serum inflammatory markers reflect the inflammatory response of the body to malignant tumors. For example, $\mathrm{C}$-reaction protein(CRP)is an acute phase protein produced by the liver after cytokines stimuli. Inflammationbased cumulative prognostic score systems were shown to be associated with levels of serum chemokines in patients with lymphoma [44]. The molecular mechanism of high degree of systemic inflammation status in 
Table 6 Survival rate and relative risk according to risk group as defined by the ICPS system

\begin{tabular}{llllllll}
\hline Risk group & No.of patients (\%) & 3 -year OS (\%) & RR $(95 \% \mathrm{Cl})$ & $P$-value & 3 -year PFS (\%) & RR (95\%Cl) & $P$-value \\
\hline ICPS $=0$ & $202(35.8)$ & 95.6 & $1.000(\mathrm{~N} / \mathrm{A})$ & $<0.001$ & 86.5 & $1.000(\mathrm{~N} / \mathrm{A})$ & $<0.001$ \\
$\mathrm{ICPS}=1$ & $144(25.5)$ & 88.2 & $2.270(1.063-4.847)$ & 0.034 & 82.3 & $1.409(0.838-2.369)$ & 0.196 \\
$\mathrm{ICPS}=2$ & $99(17.6)$ & 76.0 & $4.395(2.118-9.118)$ & $<0.001$ & 71.6 & $2.241(1.333-3.767)$ & 0.002 \\
$\mathrm{ICPS}=3$ & $119(21.1)$ & 62.2 & $8.645(4.482-16.676)$ & $<0.001$ & 54.5 & $3.957(2.518-6.216)$ & $<0.001$ \\
\hline
\end{tabular}

Abbreviations: 3Y-OS 3-year overall survival, 3Y-PFS 3-year progression-free survival, RR Relative risk, 95\% Cl, 95\% confidence limits, ICPS Inflammation-based cumulative prognostic score

malignant patients is unknown, whether these patients have abnormity of inflammatory pathways awaits future investigation. If be confirmed, these inflammation-based score systems may be useful in identifying patients for further inflammation-related mechanism research or novel anti-inflammation drug or gene target therapies.

At our lab, the normal range of CRP is $0-5 \mathrm{mg} / \mathrm{L}$, the normal range of albumin level is $>35 \mathrm{mg} / \mathrm{L}$, the normal range of lymph:mono ratio is not defined. The cut-off value of albumin level in this study is $41.5 \mathrm{mg} / \mathrm{L}$ based on ROC analysis, which falls into the normal range. Univariate analysis showed that albumin levels were positively correlated with OS and PFS, regardless of whether the cut-off value was $41.5 \mathrm{mg} / \mathrm{L}$ or $35 \mathrm{mg} / \mathrm{L}$. However, multivariate analysis with albumin level and IPI risk factors found that (data was not shown): when the cut-off

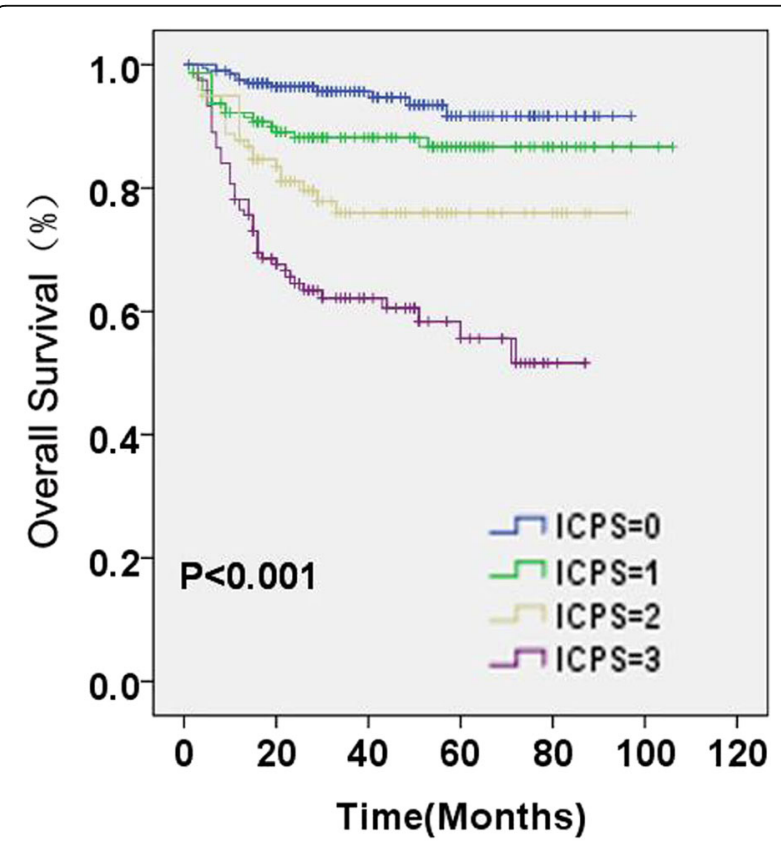

Fig. 1 Overall survival (OS) of patients based on inflammation-based cumulative prognostic score (ICPS) system. The ICPS system classified patients into four OS risk groups (ICPS $=0, I C P S=1, I C P S=2$ and ICPS = 3) value was set at $41.5 \mathrm{mg} / \mathrm{L}$, low albumin level would be an independent risk factor for poor outcomes $(P=0.002$ for OS, $P=0.004$ for PFS). When the cut-off value was set at $35 \mathrm{mg} / \mathrm{L}$, the prognostic value of low albumin level disappeared $(P=0.442$ for OS, $P=0.338$ for $P F S)$. Although albumin level $35 \sim 41.5 \mathrm{mg} / \mathrm{L}$ was in the normal range, survival of those patients were no difference with whose albumin level $<35 \mathrm{mg} / \mathrm{L}$ (3-year survival $74.7 \%$ VS.67.5\%, $P=0.220$ for OS; $69.4 \%$ VS.59.7\%, $P=0.091$ for PFS), but significantly worse than whose $\geq 41.5 \mathrm{mg} /$ L(3-year survival 74.7\% VS.92.1\%,P $<0.001$ for OS; $69.4 \%$ VS.83.8\%,P<0.001 for PFS). So we defined $41.5 \mathrm{mg} / \mathrm{L}$ as cut-off value other than $35 \mathrm{mg} / \mathrm{L}$. Most other studies of DLBCL patients used $35 \mathrm{mg} / \mathrm{L}$ as a cut-off value, which may lead to negative results. We speculate that slight decrease of serum albumin level may be caused by increase of inflammatory cytokines secretion and vascular permeability which is induced by systemic inflammatory states in lymphoma patients. While severe hypoalbuminemia may be caused mostly by malnutrition, tumor consumption, pleural or peritoneal effusion and edema, so it is independently associated with IPI risk factors. This hypothesis requires more evidence to support.

There are several limitations in this study. First, some cases chose treatment regimens without Rituximab or did not complete their course of R-CHOP treatment because the cost of Rituximab was beyond which they can afford. In order to ensure the homogeneity of treatment, these patients were excluded, which might result in a selection bias. Second, the ICPS model is based on a single-center retrospective study in South China, which has not been validated in other populations. Multicenter prospective studies are needed to determine whether it is suitable for large sample groups.

\section{Conclusions}

In summary, we have developed a novel systemic inflammatory cumulative prognostic score system independent of the IPI in DLBCL patients treated with R-CHOP therapy. The score system based on serum CRP, albumin levels and peripheral LMR, which we called the ICPS. The ICPS model can classify patients into four risk 

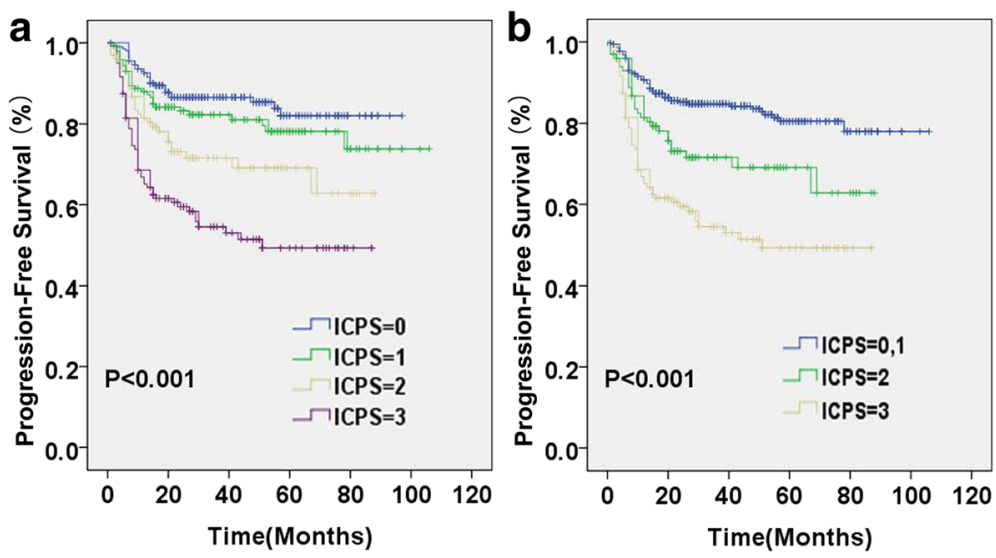

Fig. 2 Progression-free survival (PFS) of patients based on inflammation-based cumulative prognostic score (ICPS) system. a The ICPS system classified patients into four PFS risk groups (ICPS =0, ICPS $=1$, ICPS $=2$ and ICPS $=3$ ); (b) The ICPS system classified patients into three PFS risk groups (ICPS $=0-1, I C P S=2$ and ICPS $=3$ )

groups with significantly different survival outcomes. The research indicated that the degree of systemic inflammatory status was associated with clinical outcomes of patients with DLBCL receiving $\mathrm{R}-\mathrm{CHOP}$ therapy. Additional prospective multicenter studies are needed to confirm the clinical potential of the ICPS as prognostic system in patients with DLBCL. The molecular abnormity of high degree of systemic inflammation status in these DLBCL patients awaits future investigation. The ICPS model may be useful in identifying patients for further inflammation-related mechanism research or novel anti-inflammation gene or drug target therapies.

\section{Abbreviations}

CRP: C-reactive protein; DLBCL: Diffuse large B cell lymphoma; GPS: The Glasgow prognostic score; ICPS: Inflammation-based cumulative prognostic score system; IL: Interleukins; IPI: International Prognostic Index; LDH: Lactate dehydrogenase; LMR: The lymphocyte-monocyte ratio; NHLs: Non-Hodgkin's lymphomas; NLR: The neutrophil-lymphocyte ratio; OS: Overall survival; PFS: Progression-free survival; PLR: The platelet-lymphocyte ratio; PS: Performance status; ROC: Operating characteristic; TNF: Tumor necrosis factor

\section{Acknowledgements}

Not applicable.

\section{Funding}

This research was supported by National Natural Science Foundation of China (NSFC) projects (81230045) and the Ministry of Science and Technology fund of China (2012CB967003). The funding body had no role in the design of the study and collection, analysis, and interpretation of data and in writing the manuscript.

\section{Availability of data and materials}

The clinical datasets collected and/or analyzed during the current study are available from the corresponding author on reasonable request.

\section{Authors' contributions}

Data acquisition: FS, JZ, MZ, XS, ZD; data interpretation: FS, JZ, SL, JW, JH,ZD; statistical analyses: FS, JZ, SL,ZD; manuscript drafting: FS, JZ, ZZ, MZ; manuscript revision and final approval: FS, JZ, SL, ZZ, JW, JH, MZ, XS,ZD. All authors read and approved the final manuscript.

\section{Ethics approval and consent to participate}

It is a retrospective study that only involves the collection of existing clinical data and medical records. The research did not include any direct or indirect identification information of study participants. The study participant' privacy, confidentiality and anonymity was guaranteed. The rights or interests of study participants were not violated. The risk of the study participant was not greater than the minimum risk. Because of the retrospective nature of the study, the informed consent of each participant was not available. According to the guidelines for the review of major ethical issues in People's Republic of China's clinical research, this type of study meet the conditions of exemption from formal ethics approval and written informed consent.

\section{Consent for publication}

Not applicable.

\section{Competing interests}

The authors declare that they have no competing interests.

\section{Publisher's Note}

Springer Nature remains neutral with regard to jurisdictional claims in published maps and institutional affiliations.

\section{Author details}

${ }^{1}$ State Key Laboratory of Oncology in South China, Collaborative Innovation Center for Cancer Medicine, Sun Yat-sen University Cancer Center, NO.651 of Dongfeng East Road, Guangzhou 510060, China. ${ }^{2}$ Department of Pediatric Oncology, Sun Yat-sen University Cancer Center, NO.651 of Dongfeng East Road, Guangzhou 510060, China. ${ }^{3}$ Department of Biochemistry and Molecular Biology, Mayo Clinic Scottsdale, 13400 East Shea Boulevard, Scottsdale, AZ 85259, USA.

Received: 31 October 2016 Accepted: 18 December 2017

Published online: 02 January 2018

References

1. Lossos IS, Czerwinski DK, Alizadeh AA, Wechser MA, Tibshirani R, Botstein D, Levy R. Prediction of survival in diffuse large-B-cell lymphoma based on the expression of six genes. N Engl J Med. 2004;350(18):1828-37.

2. Meyer PN, Fu K, Greiner TC, Smith LM, Delabie J, Gascoyne RD, Ott G, Rosenwald A, Braziel RM, Campo E, et al. Immunohistochemical methods for predicting cell of origin and survival in patients with diffuse large B-cell lymphoma treated with rituximab. J Clin Oncol. 2011;29(2):200-7.

3. Salles G, de Jong D, Xie W, Rosenwald A, Chhanabhai M, Gaulard P, Klapper W. Calaminici M, Sander B, Thorns C, et al. Prognostic significance of immunohistochemical biomarkers in diffuse large B-cell lymphoma: a study from the Lunenburg lymphoma biomarker consortium. Blood. 2011;117(26):7070-8. 
4. Shipp MA, Ross KN, Tamayo P, Weng AP, Kutok JL, Aguiar RC, Gaasenbeek M, Angelo M, Reich M, Pinkus GS, et al. Diffuse large B-cell lymphoma outcome prediction by gene-expression profiling and supervised machine learning. Nat Med. 2002;8(1):68-74.

5. Friedberg JW. New strategies in diffuse large B-cell lymphoma: translating findings from gene expression analyses into clinical practice. Clin Cancer Res. 2011;17(19):6112-7.

6. Zhou Z, Sehn LH, Rademaker AW, Gordon LI, Lacasce AS, Crosby-Thompson A, Vanderplas A, Zelenetz AD, Abel GA, Rodriguez MA, et al. An enhanced international prognostic index (NCCN-IPI) for patients with diffuse large Bcell lymphoma treated in the rituximab era. Blood. 2014;123(6):837-42.

7. Baecklund E, Iliadou A, Askling J, Ekbom A, Backlin C, Granath F, Catrina Al, Rosenquist R, Feltelius N, Sundstrom C, et al. Association of chronic inflammation, not its treatment, with increased lymphoma risk in rheumatoid arthritis. Arthritis Rheum. 2006:54(3):692-701.

8. Loong F, Chan AC, Ho BC, Chau YP, Lee HY, Cheuk W, Yuen WK, Ng WS, Cheung HL, Chan JK. Diffuse large B-cell lymphoma associated with chronic inflammation as an incidental finding and new clinical scenarios. Mod Pathol. 2010:23(4):493-501.

9. Sagaert X, Van Cutsem E, De Hertogh G, Geboes K, Tousseyn T. Gastric MALT Iymphoma: a model of chronic inflammation-induced tumor development. Nat Rev Gastroenterol Hepatol. 2010;7(6):336-46.

10. Balkwill F, Mantovani A. Inflammation and cancer: back to Virchow? Lancet. 2001;357(9255):539-45.

11. Zhang L, Yang J, Qian J, Li H, Romaguera JE, Kwak LW, Wang M, Yi Q. Role of the microenvironment in mantle cell lymphoma: IL-6 is an important survival factor for the tumor cells. Blood. 2012;120(18):3783-92.

12. Marri PR, Hodge LS, Maurer MJ, Ziesmer SC, Slager SL, Habermann TM, Link BK, Cerhan JR, Novak AJ, Ansell SM. Prognostic significance of pretreatment serum cytokines in classical Hodgkin lymphoma. Clin Cancer Res. 2013; 19(24):6812-9.

13. Takahashi K, Sivina M, Hoellenriegel J, Oki Y, Hagemeister FB, Fayad L, Romaguera JE, Fowler N, Fanale MA, Kwak LW, et al. CCL3 and CCL4 are biomarkers for $\mathrm{B}$ cell receptor pathway activation and prognostic serum markers in diffuse large B cell lymphoma. Br J Haematol. 2015;171(5):726-35.

14. Beguelin W, Sawh S, Chambwe N, Chan FC, Jiang Y, Choo JW, Scott DW, Chalmers A, Geng H, Tsikitas L, et al. IL10 receptor is a novel therapeutic target in DLBCLs. Leukemia. 2015;29(8):1684-94.

15. Kurzrock R, Voorhees PM, Casper C, Furman RR, Fayad L, Lonial S, Borghaei $H$, Jagannath S, Sokol L, Usmani SZ, et al. A phase I, open-label study of siltuximab, an anti-IL-6 monoclonal antibody, in patients with B-cell nonHodgkin lymphoma, multiple myeloma, or Castleman disease. Clin Cancer Res. 2013;19(13):3659-70.

16. Goumas FA, Holmer R, Egberts JH, Gontarewicz A, Heneweer C, Geisen U, Hauser C, Mende MM, Legler K, Rocken C, et al. Inhibition of IL-6 signaling significantly reduces primary tumor growth and recurrencies in orthotopic xenograft models of pancreatic cancer. Int J Cancer. 2015;137(5):1035-46.

17. Drutskaya MS, Nosenko MA, Atretkhany KS, Efimov GA, Nedospasov SA. Interleukin-6 from molecular mechanisms of signal transduction to physiological properties and therapeutic targeting. Mol Biol. 2015;49(6):937-43

18. Liu FT, Jia L, Wang P, Farren T, Li H, Hao X, Agrawal SG. CD126 and targeted therapy with Tocilizumab in chronic lymphocytic leukemia. Clin Cancer Res. 2016;22(10):2462-9.

19. Rose-John S, Schooltink H. Cytokines are a therapeutic target for the prevention of inflammation-induced cancers. Recent results in cancer research Fortschritte der Krebsforschung Progres dans les recherches sur le. Cancer. 2007;174:57-66.

20. Troppan K, Deutsch A, Gerger A, Stojakovic T, Beham-Schmid C, Wenzl K, Neumeister $P$, Pichler $M$. The derived neutrophil to lymphocyte ratio is an independent prognostic factor in patients with diffuse large B-cell lymphoma. Br J Cancer. 2014;110(2):369-74

21. Cao Y, Shi YX, Chen JO, Tan YT, Cai YC, Luo HY, Qiu MZ, Cai XY, Jin Y, Sun YL, et al. Serum C-reactive protein as an important prognostic variable in patients with diffuse large B cell lymphoma. Tumour Biol. 2012;33(4):1039-44.

22. Li ZM, Huang JJ, Xia Y, Sun J, Huang Y, Wang Y, Zhu YJ, Li YJ, Zhao W, Wei WX, et al. Blood lymphocyte-to-monocyte ratio identifies high-risk patients in diffuse large B-cell lymphoma treated with R-CHOP. PLoS One. 2012;7(7):e41658.

23. Nakamura K, Nishida T, Haruma T, Haraga J, Omichi C, Ogawa C, Kusumoto T, Seki N, Masuyama H, Hiramatsu Y. Pretreatment platelet-lymphocyte ratio is an independent predictor of cervical cancer recurrence following concurrent chemoradiation therapy. Mol Clin Oncol. 2015;3(5):1001-6.

24. Zhang WW, Liu KJ, GL H, Liang WJ. Preoperative platelet/lymphocyte ratio is a superior prognostic factor compared to other systemic inflammatory response markers in ovarian cancer patients. Tumour Biol. 2015;36(11):8831-7.

25. Liu YL, Lu Q, Liang JW, Xia Y, Zhang W, Hu BQ, Shang FF, Ji YR, Wang J, Wang Q, et al. High plasma fibrinogen is correlated with poor response to trastuzumab treatment in HER2 positive breast cancer. Medicine. 2015;94(5):e481.

26. Wen J, Yang Y, Ye F, Huang X, Li S, Wang Q, Xie X. The preoperative plasma fibrinogen level is an independent prognostic factor for overall survival of breast cancer patients who underwent surgical treatment. Breast. 2015;24(6):745-50

27. Zhu LR, Li J, Chen P, Jiang Q, Tang XP. Clinical significance of plasma fibrinogen and D-dimer in predicting the chemotherapy efficacy and prognosis for small cell lung cancer patients. Clin Trans Oncol. 2016:18(2):178-88.

28. Kobayashi T, Kawakamil M, Hara Y, Shioiri S, Yasuno M, Teruya M, Kaminishi M. Combined evaluation of the Glasgow prognostic score and carcinoembryonic antigen concentration prior to hepatectomy predicts postoperative outcomes in patients with liver metastasis from colorectal cancer. Hepato-Gastroenterology. 2014;61(133):1359-62.

29. Dreanic J, Dhooge M, Brezault C, Mir O, Chaussade S, Coriat R. A prognostic indicator of survival in metastatic colorectal cancer patients in the era of molecular-targeted agents: the modified Glasgow prognostic score. Oncology. 2014;86(1):44-5.

30. Gioulbasanis I, Pallis A, Vlachostergios PJ, Xyrafas A, Giannousi Z, Perdikouri IE, Makridou M, Kakalou D, Georgoulias V. The Glasgow prognostic score (GPS) predicts toxicity and efficacy in platinum-based treated patients with metastatic lung cancer. Lung Cancer. 2012;77(2):383-8.

31. Jafri SH, Shi R, Mills G. Advance lung cancer inflammation index (ALI) at diagnosis is a prognostic marker in patients with metastatic non-small cell lung cancer (NSCLC): a retrospective review. BMC Cancer. 2013;13:158.

32. Vardiman JW. The World Health Organization (WHO) classification of tumors of the hematopoietic and lymphoid tissues: an overview with emphasis on the myeloid neoplasms. Chem Biol Interact. 2010;184(1-2):16-20.

33. Antonioli L, Blandizzi C, Pacher P, Hasko G. Immunity, inflammation and cancer: a leading role for adenosine. Nat Rev Cancer. 2013;13(12):842-57.

34. Grivennikov SI, Greten FR, Karin M. Immunity, inflammation, and cancer. Cell. 2010;140(6):883-99.

35. Karin M, Greten FR. NF-kappaB: linking inflammation and immunity to cancer development and progression. Nat Rev Immunol. 2005;5(10):749-59.

36. Lin WW, Karin M. A cytokine-mediated link between innate immunity, inflammation, and cancer. J Clin Invest. 2007;117(5):1175-83.

37. Kim Y, Kim SJ, Hwang D, Jang J, Hyun SY, Kim YR, Kim JS, Min YH, Cheong JW. The modified Glasgow prognostic scores as a predictor in diffuse large B cell lymphoma treated with R-CHOP regimen. Yonsei Med J. 2014;55(6):1568-75.

38. Liao F, Hsu YC, Kuo SH, Yang YC, Chen JP, Hsu PN, Lin CW, Chen LT, Cheng $A L$, Fann CS, et al. Genetic polymorphisms and tissue expression of interleukin-22 associated with risk and therapeutic response of gastric mucosa-associated lymphoid tissue lymphoma. Blood Cancer J. 2014;4:eXX.

39. Lech-Maranda E, Baseggio L, Bienvenu J, Charlot C, Berger F, Rigal D, Warzocha K, Coiffier B, Salles G. Interleukin-10 gene promoter polymorphisms influence the clinical outcome of diffuse large B-cell lymphoma. Blood. 2004;103(9):3529-34.

40. Cunningham LM, Chapman C, Dunstan R, Bell MC, Joske DJ. Polymorphisms in the interleukin 10 gene promoter are associated with susceptibility to aggressive non-Hodgkin's lymphoma. Leuk Lymphoma. 2003:44(2):251-5.

41. Qian C, Liu XY, Prieto J. Therapy of cancer by cytokines mediated by gene therapy approach. Cell Res. 2006;16(2):182-8.

42. Ojima T, Iwahashi M, Nakamura M, Matsuda K, Nakamori M, Ueda K, Naka T, Ishida K, Primus FJ, Yamaue H. Successful cancer vaccine therapy for carcinoembryonic antigen (CEA)-expressing colon cancer using genetically modified dendritic cells that express CEA and T helper-type 1 cytokines in CEA transgenic mice. Int J Cancer. 2007;120(3):585-93.

43. Dranoff G. Cytokines in cancer pathogenesis and cancer therapy. Nat Rev Cancer. 2004:4(1):11-22.

44. Kim SJ, Ryu KJ, Hong M, Ko YH, Kim WS. The serum CXCL13 level is associated with the Glasgow prognostic score in extranodal NKT-cell lymphoma patients. J Hematol Oncol. 2015;8:49. 